\title{
La Médecine Vétérinaire Chez Les Arabes \\ Depuis Le VIième jusqu'au IXième Siècle de l'Hégire \\ 'Gehane Heshmat
}

\section{Summary}

The reign of the mamlūk between $\mathrm{VI}^{\text {th }} \mathrm{h} / \mathrm{XII}{ }^{\text {th }}$, and $\mathrm{IX}^{\text {th }} / \mathrm{XV}^{\text {th }}$ after J.C., was very rich in military exercises, ceremonials, wars. Veterinary medicine took an important role at that time, the article is reporting on that subject.

According to the research we can tell that they were three kinds of medicine at that time:

a- Preventive medicine, based on hygiene, cleanliness of animals, stables and food given to animals.

b- Curative medicine, which is divided into two branches: operation like castration, embryotomie, and other. Second branch where arabic veterinary used incisions, drops, infusions, oitments, enema and other methods to cure diseases.

c- Popular medicine, like using coran in curing animals or suspending amulets to animals as to provide illness and evil eye.

We can also add that people who worked in iron (forge), used to practice slightly veterinary medicine.

We also notice that animals during major operation were not taken anesthesia like opium, in reverse veterinary used to make them inconscious during treatments.

•ProfesseuriIslamologue à l'Institut d'Hôtellerie et de Tourisme, Sheraton Heliopolis. 
L'immense intérêt que les arabes portèrent au cheval est perceptible à travers un grand nombre de traités et d'ouvrages dont une litérature savante qui concerne l'hippiatrie (al-bayțara), et la médecine des chevaux. La période qui se classe entre VIième de l'hégire/XIIième siècle, et celle du IXième/XVième aprés J.C., fut abondante en activité équestre, particulièrement chez la dynastie mamlūke. Ce fut aussi le cas dans le domaine vétérinaire sur lequel on jettera la lumière.

On reconnait un certain nombre de célèbres hippiatres qui composèrent des traités vétérinaires, à titre d'exemple, Ahmad Hasan Ibn al-Ahnaf 605/ 1209 qui composa " kitāb al-bayțara", Baktūt al-Ramāh 711/1311, qui rédigea un ouvrage intitulé "Kitāb al-furūsīya wa 'ilāğ al-hayl", citons de même le traité d'Abī Bakr Ibn Badr al-Dīn Ibn al-Bīțār, ${ }^{2}$ 741/1340 écrit à la demande du sultan mamlūk al-Nāşir Muhammad Ibn Qalāwūn, intitulé "Kāmil al-şina ātyin". ${ }^{3}$

Quelles sont les maladies les plus répandues à cette époque?

Les vétérinaires à cette époque rapportent que les maladies qui attaquent les animaux sont aussi ceux qui attaquent l'être humain. Ceci s'applique en général pour les remèdes. ${ }^{4}$ A titre d'exemple les maladies cutanées le vitiligo caractérisé par l'apparition des tâches blanches sur la bête ${ }^{5}$.On cite aussi les

\footnotetext{
${ }^{1}$ Ahmad 'Abd al-Rāziq, al-Hadāra al-islāmīya fĩ al-'uşūr al-wusța, al-'alūm al-'aqlīya , Dār alFikr al-' arabī, al-Qāhira, 1991,p. 263; Nabīl 'Abd al-'Azīz al-Hayl wa riyādatihā fî 'aşr şalāţīn al-mamālīk, Le Caire, 1976, p.12.

${ }^{2}$ En arabe البيطـــ al-bayțarī, celui qui pratique la médecine des animaux. Ce terme est tiré d'un terme grec "hippiatros" ; Hasan al-Baša, al-Funūn al-islāmīya wal wazā'if 'ala al-'atār al'arabīya, I, Dār al-Nahda al-'Arabīya, Le Caire, 1965,p.325.

${ }^{3}$ Abū Bakr Ibn Badr al-Dīn Ibn al-Mundir al-Bayțār, Hippologie et médecine du cheval en terre d'islam au XIVième siècle, Le traité des deux arts en médecine vétérinaire, Traduction de l'arabe de M.M. Hakimi, sous la direction de C.Degueurce et avec la participation de F.Vallat et A.Vernay Nouri, editions errance, Paris, 2006, introduction; 'Abd al-Rāziq, alHadāra, p.263.

${ }^{4}$ Voir Abī Bakr Ibn al-Badr Ibn al-Bịțār, Kāmil al-şena āâ̄n fī al-bayțara wal zardaqa, réalisé par 'Abd al-Rahmān Ibrīq, Alep, 1993, première partie. La première partie de ce livre contient tous les maladies relatives aux animaux précisement les chevaux.

${ }^{5}$ Abū Bakr, Le traité, pp.57-60;
} 
déformations congénitales, ${ }^{6}$ à titre d'exemple déformation du dos,(P1.III) ${ }^{7}$ les maladies inguérissables, ${ }^{8}$ comme "al-Hanām alrațb", tumeur du cerveau, ${ }^{9}$. D'autres maladies comme celles des organes génitaux femelles et mâle, ${ }^{10}$ les maladies des articulations, 11 les maladies infectieuses et contagieuses comme la fièvre charbonneuse, ${ }^{12}$ le tétanos, la rage, et la peste. ${ }^{13}$ En bref, les troubles généraux, maladies de l'appareil digestif de l'appareil respiratoire et circulatoire (cardiaque), les maladies des yeux, de la tête, et les affections des membres. ${ }^{14}$

\section{Soignement de Maladies}

L'anatomie de la bête était considérée phase essentielle pour un vétérinaire quant à la guérison des maladies. Les vétérinaires arabes exercaient la chirurgie des animaux en se basant sur les connaissances anatomique. ${ }^{15}$ (Pls.I, II). ${ }^{16}$ Suivant les sources et les références, on peut affirmer l'existence de trois genres de médecine. médecine préventive, curative et populaire.

\section{Médecine Préventive}

Il est intéréssant de savoir qu'il existait une écurie, sorte d'hôpital pour les animaux fatigués ou malades. ${ }^{17}$ L'hygiène était

${ }^{6}$ al-Malț al-Ašrafī, al-Bayțara mimā ğama uh wa tasrīfuh, kitāb al-mug̉nī, ms. 745, fls, $12,13,15$.

7al-Hasan Ibn al-Ahnaf, Muhtaşar Kitāb al-Bayțara, Institut des Manuscrits Arabes ms.223 tibb, fol. 76 .

${ }^{8}$ Ibn al-Ahnaf, Muhtaşar,, ms. 223 țibb, fol. 75.

${ }^{9}$ Anonyme, Kitāb al-bayțara wa mā yat’alaq bil dawāb, ms. 45, rédigé 902/1496, Université du Roi Sa ud, fol.76

${ }^{10}$ Abū Bakr, Le traité, p.77,

${ }^{11}$ Abū Bakr, Le Traité, p.84.

${ }^{12}$ Maladie infectieuse des végétaux produisant une poussière noire; Cf. Dictionnaire Le Robert d'aujoud'hui, Paris, 1993, p.159.

${ }^{13}$ L.Moulé, Histoire de la médecine vétérinaire, Imprimerie Maulde-Doumenc, Paris,1800, p.5.

${ }^{14}$ Gabrielle D'Houdain, Histoire de la saignée vétérinaire, Thèse de doctorat pour le doctorat vétérinaire, Faculté de Médecine de Créteil, Paris, 2001, p.10.

${ }^{15}$ Bouazza Kherrati, Histoire de la médecine vétérinaire, extrait publié par le Cabinet Vétérinaire El Naweras, 1998, Alger, p.1.

${ }^{16}$ Rania Sayyed, Anatomy of The Horse in the $15^{\text {th }}$ Century, http://www.muslimheritage.com/, d'aprés l'internet.

${ }^{17}$ Nabīl, al-Hayl, p.103. 
de premier ordre. Tout d'abord l'écurie devrait être bien aérée et vaste. Le palfrenier était chargé du nettoyage de l'écurie, de l'animal, ainsi que les pieds. Par exemple comme mesures d'hygiène le palfrenier devait nettoyer le corps du cheval chaque matin, ${ }^{18}$ et faire attention de couvrir le par terre de l'écurie en sable fin et bois afin de combattre les insectes comme le ver du tapis. De même il fallait faire un trou au dessous des pieds de l'animal pour recevoir l'urine, afin de le garder propre. ${ }^{19}$

A cette époque les sultans mamlūk conseillait les palfreniers "al-suwās de nettoyer l'orge de poussière, de tamiser le grain pour éviter l'endommagement des dents, ${ }^{20}$ la luzerne séche et hachée, le fourrage vert doit être distribué par petites quantités et l'eau à volonté $^{21}$ sauf si l'animal est essouflé. ${ }^{22}$

Autre rôle du palfrenier "al-sāȳ̄s" c'était la connaissance à fond des animaux leurs douleurs leurs maladies, ce qui leurs convenait comme médicaments, onguents. Il était indispensable que le palfrenier soit né parmi les maîtres palfreniers, qu'il devrait respecter. ${ }^{23}$

Suivant les traités d'hippiatrie, comme mesure de précautions pour les animaux, il était recommandé d'entrainer les chevaux au début du printemps pour éviter les périodes de chaleur qui sont insupportables à l'animal, ou au commencement de l'automne, pour se mettre à l'abri du froid glacial qui fatigue l'animal. ${ }^{24}$

$\mathrm{Au}$ témoignage des sources, une autre méthode préventive qui permettait de nettoyer les intestins de l'animal tel que le cheval, était de placer les chevaux au vert dans des endroits nommés

\footnotetext{
18 ‘Abd al-Qādir al-Ğazā'irī, kitāb ‘aqd al-’ağyād fī al-şafanāt al-ğīyād, Syrie, 1393, p.272.

${ }^{19}$ Nabīl 'Abd al- 'Azīz, al-Hayl, p.31.

${ }^{20}$ Tāğ al-Dīn al-Subkī, Mu ${ }^{e} \overline{1} d$ al-ni ${ }^{\circ}$ am wa mubīd al-niqam, réalisé par Muhammad 'Alī alNağār , Le Caire, 1948, pp.144, 145; Nabīl, al-Hayl, p.25.

${ }^{21}$ M.Mousnier, Les animaux malades en europe occidentale, VIième-XIXiè siècle, Presses universitaires du Mirail, 2005, p.46.

${ }^{22}$ al-Ašrafì, al-Muġnī, fol.16; Nabīl, al-Hayl, p.25.

${ }^{23}$ Nabīl, al-Hayl, p.123

${ }^{24}$ al-Ğazā'irī, ‘aqd, p.276; Nabīl, al-Hayl, p.24.
} 
(marābit al-hayl). e procédure consistait à laisser les chevaux au printemps paître en liberté dans des champs d'orges et de trèfles, afin que l'usage de cette nourriture rafraîchissante les délassât de leur fatigue, et puisse nettoyer leur intestin et leurs procurer des forces nouvelle. ${ }^{25}$ Au dire d'al-Maqrīzī les champs de trèfles (marābit al-hayl) se situaient hors du Caire à proximité de Giza comme la ville d'Usīm. ${ }^{26}$

Le vétérinaire devrait éviter de soigner des animaux de médication échauffante en saison chaude, ni de médication froide en saison froide. Il est en de même pour les opérations chirurgicales, il faut se garder d'ouvrir chirurgicalement un animal en hiver et par grand froid, à l'inverse ne pas ouvrir un animal chirurgicalement aux plus fortes chaleurs. ${ }^{27}$

La saignée dont on parlera ci-dessous était aussi considérée une méthode préventive, à la suite d'une fourbure, ou aprés une indigestion d'orge. ${ }^{28}$

\section{Médecine Curative}

A cette époque, le muhtasib (le préfet du marché) ne permettait la pratique de la médecine vétérinaire qu'a la suite d'un examen spécialisé. $^{29}$

Les hippiatres de cette époque suivaient certains protocoles, faute de nuire aux animaux. On lit chez Abū Bakr Ibn al-Bīțār, à propos des conseils donnés aux hippiatres concernant les soins et les remèdes. Le premier conseil est de respecter ses professeurs et les honorer. Aux dires d'Ibn al-Bīțār "Si l'on se remet à vous pour un conseil sur un animal, il faut témoigner en toute vérité, sans détour. Il rapporte aussi qu'une maladie sans remède possible comme la boiterie chronique, le vitiligo, il ne faut pas se lancer dans les

\footnotetext{
${ }^{25}$ Ibn Iyās, Badā'i ${ }^{`}$ a al-zuhur fĩ waqā' 'i al-duhur, lière impression, Būlāq, II, p.124.

${ }^{26}$ Taky al-Dīn al-Maqrīzī, al-Suluk li ma rifat duwal al-muluk, réalisé par Muhammad Musțafa zīyāda et Sa ${ }^{\top} \bar{d}{ }^{~ ' A b d ~ a l-F a t t a ̄ h ~ ' A s ̌ u ̄ r, ~ L e ~ C a i r e, ~ 1956-1973, ~ I V, ~ 1 e ̀ r e ~ p a r t i e, ~ p .120 ~}$

${ }^{27} \mathrm{Abū}$ Bakr, Le traité, p.88.

${ }^{28}$ Mousnier, Les animaux, p.40.

${ }^{29}$ Ibn al-Uhuwwa, Ma`ālim al-qurba fī ahkām al-hisba, Rubin Lévi, Cambridge, 1937; ‘Abd alRāziq, al-Hadāra, pp.262,263.
} 
traitements car ces maladies sont inguérissables. En outre il faut connaître les médicaments utilisés pour le traitement des maladies, leurs forces, et leurs indications. ${ }^{30}$

\section{Les Soins Chirurgicaux}

Quels sont les instruments utilisés dans les opérations chirurgicales? Suivant les chercheurs les outils les plus utilisés sont une lancette ${ }^{31}$, un petit bistouri, le rasoir, les fils, l'aiguille, la rénette $^{32}$ et les tricoises ${ }^{33}$.

Parmi les soins chirurgicaux on cite: les incisions, la scarification, (P1.IV). Ainsi lit-on chez Ibn al-Ahnaf que pour guérir les maladies de gorge et d'autres, le vétérinaire scarifier l'animal au front sous la langue, il laissait l'animal lêcher le sang. Peu aprés l'animal est privé de nourriture et d'eau jusqu'à quelques heures. ${ }^{34}$ Le débridement des plaies, l'extraction des dents, la castration $^{35}$ ainsi que la saignée et d'autres font aussi partie des soins chirurgicaux. ${ }^{36}$

L'extraction des dents ou molaires surnuméraires était importante car elle empêchait une bonne mastication des aliments et par conséquent l'animal devenait chétif. $(\mathrm{Pl} . \mathrm{V}){ }^{37}$

La Saignée. Que-est ce que la saignée?

La saignée est l'ouverture d'une veine afin de retirer le sang qui stagne à l'endroit de l'affection ${ }^{38}$

\footnotetext{
${ }^{30}$ Abū Bakr, Le traité, pp.88,89.

${ }^{31}$ Petit instrument de chirurgie qui était utilisé pour la saignée, la vaccination et l'incision des abcés, Dictionnaire Petit Larousse, Canada. 1990, p.559.

${ }^{32}$ Outil tranchant pour tailler le sabot du cheval, Dictionnaire Larousse,p. 812.

${ }^{33}$ Moulé, Histoire, p.22, Gabrielle, Histoire de la saignée, p56; tricoises, veut dire tenailles, Cf. Dictionnaire Larousse, p.986.

${ }^{34}$ Ibn al-Ahnaf, Muhtaşar, fol.133

${ }^{35}$ Moulé, Histoire, p. 22.

${ }^{36}$ Mousnier, Les animaux, p.51.

${ }^{37}$ Ibn al-Ahnaf, Muhtaşar kitāb al-bayțara, , version qui se trouve à Dār al-Kutub al-Maşrīya, ms. 167 , fol.17.

${ }^{38}$ Abū Bakr, Le traité,p.89; Dictionnaire Larousse, p.867; Gabrielle, Histoire de la saignée, p. 15 .
} 
Le vétérinaire $A b u \overline{~ B a k r}$, rapporte qu'avant la pratique de la saignée, il fallait être en mesure de savoir quelle quantité de sang il faut prélever, car dit-il l'état de l'animal s'aggraverait si l'on soutire plus de sang. La quantité de sang une à deux livres ou une à deux onces. ${ }^{39}$ Suivant le vétérinaire al-Ašrafĩ trois litres pour un cheval vigoureux et deux litres pour un petit cheval. ${ }^{40}$ La quantité de sang retirée, aux poulains, aux jeunes chevaux, aux mulets et aux ânes, devrait être basse à cause de la petitesse du corps, quant aux chevaux accomplis, chevaux de grande constitution la quantité de sang à retirer serait plus élevée. ${ }^{41}$

L'opération de la saignée s'appliquait sur maintes endroits. A titre d'exemple les veines jugulaires ${ }^{42}$ pour mélanose, ${ }^{43}$ et l'ensemble des maladies concernant l'encolure. Saigner pour l'affection des membres antérieures, comme épaule meurtrie, inflammation du garrot ${ }^{44}$. Saigner les membres postérieures lors des douleurs dans la région des reins, des hanches. ${ }^{45}$ La poction du nez dans le cas d'asthme, la poction du sabot dans le cas d'érysipèle ${ }^{46}$. On cite aussi la saignée des veines saphènes,${ }^{47}$ et des veines pectorales et d'autres. ${ }^{48}$

Faut-il savoir que certains opérations exigeaient la présence de deux vétérinaires à la fois. Comme celle de l'infection de la poitrine. A l'aide du bistouri le vétérinaire applique un trou à la poitrine qu'il laisse ouvert pour la sortie du pus, en même temps

\footnotetext{
${ }^{39}$ Mesure de poids valant environ 27,25 g,ou une livre douze;Dictionnaie petit Larousse, p.681.

40 al-Ašrafī, al-Muginī, fol.19.

${ }^{41}$ Abū Bakr, Le traité, pp.89,90; Gabrielle, Histoire de la saignée, p;.42.

${ }^{42}$ Veines qui appartiennent à la gorge, au cou; Dictionnaire Larousse, p.549.

${ }^{43}$ Accumulation localisée de pigment mélanique dans les tissus, Dictionnaire Larousse, p.613.

${ }^{44}$ Région du corps des grands quadrupèdes surmontant les épaules et délimitée par l'encolure le dos et le plat des épaules, Petit Larousse, p.451.

${ }^{45}$ Gabrielle, Histoire de la saignée, p.40.

${ }^{46}$ Maladie infectieuse, caractérisée par une inflammation douleureuse de la peau; Dictionnaire Larousse, p.385.

${ }^{47}$ Se dit des deux veines qui collectent le sang des veines superficielles du membre inférieur; Dictionnaire Larousse, p.873.

${ }^{48}$ Abū Bakr, Le traité, p.89
} 
l'autre vétérinaire applique la fumigation comme antiseptique à l'animal (Pl.VI) ${ }^{49}$.

Ainsi lit-on que pour la guérison d'u animal qui souffre des douleurs aux jarrets, il faudrait passer une corde, longue et fine depuis la tête jusqu'au au delà des jarrets, au moyen du bistouri ouvrir l'endroit désigner et laisser le sang s'écouler, saigner de même les jarrets, ensuite appliquer du bitume dilué dans de la cire et couvrir les endroits saignés avec un morceau de lin. ${ }^{50}$

Il faut savoir que généralement, à la suite des opérations de la saignée ou d'autres l'animal était privé de la nourriture et du boire, et on le mettait au repos. ${ }^{51}$

Les opérations étaient aussi un moyen pour la guérison des maladies ophtalmologiques comme celle de l'onglet "al-zafra" sorte de corps qui a la forme d'une oiseau qui prend naissance à l'angle de l'œil, ensuite se trouve au milieu de l'œil. ${ }^{52}$ Ainsi pour ôter l'onglet, le vétérinaire ligotait le cheval aux pieds, et posait un coussin sous sa tête, il fait apparaitre la lésion "al-zafra" à l'extérieur et l'accroche avec un fil monté sur une aiguillle, et il l'incise avec un rasoir. Par la suite il y applique du sel dans l'œil et couvre la tête de l'animal d'un morceau de lin. ${ }^{53}$

Un autre exemple d'opération chirurgicale, celle de la castration. En principe la castration est désapprouvée par les théologiens et les hommes de science. Cependant on en avait recours pour la guérison de certaines maladies comme les troubles cérébraux, la rage et quelques maladies rénales. Au dire d'Abū Bakr le ministre du calife abasside al-Mu'ațasim avait un cheval aux sabots fins, et lorsqu'il l'a castré les sabots se sont renforcés. ${ }^{54}$

\footnotetext{
${ }^{49}$ Ibn al-Ahnaf, Muhtaşar, fls.230-231.

${ }^{50}$ al-Ašrafì, al-Muğnī, fol.16.

${ }^{51}$ Gabrielle, Histoire de la saignée, pp.45,46.

${ }^{52}$ Abū Bakr, Le traité, p.62.

${ }^{53}$ al-Ašrafī, al-Muğnī, ms. 745, fol.31; Cf. Abū Bakr, Le traité p.106.

${ }^{54}$ Abū Bakr, Le traité, p.153.
} 
A cette époque il existait plusieurs méthodes pour castrer l'animal rapporte le vétérinaire Abū Bakr. La castration par feu, par écrasement, par incision et par extraction. Suivant Abū Bakr, la méthode la moins dangereuse est celle de la castration par feu. Le cheval couché sur le dos les membes en l'air. Il faut en suite tenir les testicules et les attacher solidement à leur origine d'une cordelette, inciser le scrotum au cautère aiguisé, découvrir les testicules de toutes leur enveloppes les tenir à leur origine à l'aide d'un casseau constitué d'un rameau de dattier puis couper à l'origine avec la lame de cautère. Ensuite appliquer des gouttes de la poix à l'extrémité des vaisseaux pour empêcher l'écoulement de sang, tout en maintenant l'extrémité jus'qu'à la coagulation. Retirer la cordelette et oindre les plaies d'huile de sel et d'ail. ${ }^{55}$

Autre pratique chirurgicale, c'est l'embryotomie. On pratiquait l'embryotomie dans le cas de la malformation de l'embryon, qui pourrait nuire à la jument. Dans ce cas, Ibn al-Ahnaf rapporte que le vétérinaire devait introduire la main dans la matrice de la jument et faire suffoquer l'embryon, ensuite le tirer de la matrice. Cette opération était suivie du soignement de la matrice de la jument avec de l'huile et du sel. Ensuite le vétérinaire bouchait la vulve avec de la laine trempée dans l'huile. Une seconde méthode pour l'embryotomie, le vétérinaire plongeait les mains dans l'huile ensuite à l'aide d'un instrument il extrayait l'embryon mort de la matrice, cette opération était suivie par le soignement de la matrice, ensuite la jument était mise au repos. (Pl.VII). ${ }^{56}$

En dépit des soins chirurgicaux, les vétérinaires avaient recours à d'autres méthodes curatives. Prenons comme exemple, les breuvages, cataplasmes, ${ }^{57}$ les collyres, ${ }^{58}$ les infusions, la cautérisation, la fumigation dite en arabe "les teintures.

\footnotetext{
${ }^{55}$ Abū Bakr, Le traité, p.153.

${ }^{56}$ Ibn al-Ahnaf, Muhtaşar, fol.89.

${ }^{57}$ Abū Bakt, Le traité, p. 177.

${ }^{58}$ Ibn al-Ahnaf, Muhtaşar, ms.167, fol.192
} 
D'autres méthodes comme les poudres dermatologiques, ${ }^{59}$ pommades, onguents, ${ }^{60}$ pâtes dermiques, les plantes fraîches broyées et placées entre deux compresses, les lavages ${ }^{61}$ et les suppositoires. ${ }^{62}$

Prenons comme exemple la cautérisation, qui était une méthode employée seule ou avec d'autres moyens curatifs. La cautérisation c'est l'action de brûler au moyen d'un cautère pour des fins thérapeutiques. Suivant le célèbre vétérinaire du sultan alNāşir Muhammad Ibn Qalawūn, il existe plusieurs cautères relatifs à une diversité de maladies, cautère pour le cancer autre lombaire, un troisième pour l'épaule, ainsi de suite ${ }^{63}$. On lit de même dans l'ouvrage d'al-Ašrafî, à propos d'une cautérisation d'un cancer sur le pied d'un cheval. ${ }^{64}$

Ibn al-Ahnaf rapporte que la guérison d'un surplus d'os se faisait par le biais de la cautérisation, l'endroit rasé et cautérisé, ensuite, on lavait l'endroit avec l'eau de rose, leau chaude et de l'huile. Cette opération se répétait chaque deux jours, jusqu'à que la poussée du poil (Pl. VIII) .

A cette époque les vétérinaires connaissaient aussi les emplâtres, les attelles en rameaux de palmier ou en bois afin d'empêcher le mouvement de l'animal, ${ }^{66}$ (P1.IX) ainsi que les emplâtres en bois pour guérir les fractures. Parfois aussi le traitement exigeait l'emploi du cautère, ceci en dépit des cataplasmes comme la sève de pin, l'aloés, la colle de poisson, qui étaient bouillis en parts égales et épaissis ${ }^{67}$ avec l'asphodèle ${ }^{68}$.

\footnotetext{
${ }^{59}$ Abū Bakr , Le Traité, p.180

${ }^{60}$ Al-Ašrafī, al-Muġnī, fol.45.

${ }^{61}$ Anonyme, al-Bayțara al-rūmīya, manuscrit no. 1334 , Dār al-Kutub al-Masrīya, fol. 156.

62 Mousnier, Les animaux, p.50.

${ }^{63}$ Abū Bakr, Le traité, pp.178, 179.

${ }^{64}$ Al-Ašrafī, al-Muğnī, fol. 22.

${ }^{65}$ Ibn al-Ahnaf, Muhtaşar, fol.267.

${ }^{66}$ Ibn al-Ahnaf, Muhtaşar, fls. 161, 162.

${ }^{67}$ Abū Bakr, Le traité, p. 125.

${ }^{68}$ Plante bulbeuse à fleurs blanches, Dictonnaire Larousse, p.91.
} 
Les remèdes employées par les vétérinaires arabes comprenaient les traitements cutanés comme les maladies de la peau qui selon Abū Bakr, comptaient vingt-quatre, comme la gale, l'alopécie, les blessures due au métal, aux armes, les morsures de vipère et d'autres. ${ }^{69}$ A titre d'exemple Ibn al-Ahnaf rapporte que le traitement de la chute des cheveux chez le dromadaire se faisait par le biais de la graisse de porc sinon celle de la vache. Il fallait étaler la graisse sur les lésions. (P1.X). ${ }^{70}$

Les vétérinaires ont aussi appliqué d'autres remèdes comme la fumigation cité là haut, par exemple on traitait le ballonnement du ventre de l'animal en le faisant sentir du miel dilué dans d'autres ingrédients, ceci aprés avoir nettoyer l'anus des excréments, par la suite on le faisait marcher pendant une heure. ${ }^{71}$

En dépit de la fumigation, les vétérinaires arabes usaient les collyres $^{72}$ et les onguents. Ainsi Abū Bakr avance une recette pour l'albugo de l'œil. Il fallait prendre une infusion d'orge la mélanger à du beurre fondu et l'appliquer à l'œil. ${ }^{73}$

Voici une recette pour redonner de la force à un taureau qui ne parvient pas à se lever, le vétérinaire courvrait le sol de réglisse moulue ensuite il faisait passer le corps du taureau sur (Pl.XI). ${ }^{74}$

Pour les brûlures, on utilisait de l'eau de rose locale, du camphre et du blanc de plomb, ou bien des coquilles d'escargot brulées et réduites en poudre, afin de l'étaler sur les brûlures. ${ }^{75}$ Pour les morsures de scorpion, rapporte, on faisait boire à l'animal une quantité équivalente d'eau à une demi once de thériaque, qui se compose du suivant: froment romain, graines d'olivier, aristoloche

\footnotetext{
${ }^{69}$ Abū Bakr, Le traité, pp.57,58.

${ }^{70}$ Ibn al-Ahnaf, Muhtaşar, fol. 168; Quand les sciences parlent arabe,Centre français de culture et de coopération, Le Caire, Musée d'Art Islamique, 2003, p.64.

${ }^{71}$ Al-Ašrafì, al-Muğnī, fol.30.

${ }^{72}$ Ibn al-Ahnaf, Muhtaşar, fol. 192.

${ }^{73}$ Abū Bakr, Le traité, p. 175

${ }_{74}$ Quand les sciences parlent arabe, p.139.

${ }^{75}$ Abū Bakr, Le traité, p.99.
} 
longue $^{76}$ et myrrhe ${ }^{77}$ en parts égales, il fallait moudre le tout le cuire dans du miel filtré et l'instiller dans les narines de l'animal, et deux dixième avec du sirop de bois d'aigle. ${ }^{78}$

\section{La question qui se pose maintenant, y a t-il d'autres personnes outre que les vétérinaires qui pratiquaient la médecine curative?}

Le chercheur contemporain Aly Mazahéri dans son ouvrage intitulé "La vie quotidienne des musulmans au moyen âge" parlant des bazars des forgerons, il dit:"C'étaient des milliers d'ouvriers noricis par le charbon, qui s'agitaient. Les uns, un peu vétérinaires, ferraient les bêtes de somme, savaient réduire une fracture, pratiquer la saignée, châtrer les animaux et connaissaient les $<$ trois cent vingt maladies du cheval $>.{ }^{79}$

\section{Medecine Populaire}

A côté d'une médecine savante, la médecine populaire était aussi une pratique courante chez les vétérinaires arabes à cette époque. Celle-ci était pratiquait par les vétérinaires eux mêmes. Ainsi lit-on dans l'ouvrage du vétérinaire Ibn al-Ahnaf "Muhtsar kitāb al-Bayțara", que pour les accouchements difficiles de la bête on lisait les versets suivant: ${ }^{80}$

$$
\text { " ألم ير الذين كفروا أن السمو ات و الأرض كانتا رتقا فقتقنهما" }
$$

"Ceux qui ont mécru, n'ont-ils pas vu que les cieux et la terre formaient une compacte? Ensuite Nous les avons séparés" Surate XXI, verset 30 .

\footnotetext{
${ }^{76}$ Les racines sont riches en acide aristolochique (cancérigène pour les reins et l'estomac) contiennent une huile essentielle. Au Maroc elle est traditionellement utilisée contre les palpitations aortiques, les constipations et les affections digestives. On lui connait aussi des vertus diurétiques, $\mathrm{Cf}, \mathrm{Abū}$ Bakr, Le traité, p.194.

${ }^{77}$ La plante contient $60 \%$ d'une résine d'alcool et d'acides triterpéniques, $40 \%$ d'une gomme d'arabinose et de galactose. Utilisée principalement localement en onguent, elle est utilisée aussi pour soigner les affections nerveuses , Cf. Abū Bakr, Le traité, p.204.

${ }^{78}$ Abū Bakr,Le traité, p.99.

${ }^{79}$ Aly Mazahérī, La vie quotidienne des musulmans au moyen age Xième au XIIIième siècle, Hachette, Paris, 1951,p.197.

${ }^{80}$ Ibn al-Ahnaf, Muhtaşar, ms. 223, fol. 169
} 
Cette idée est aussi renforcée par le traité d'Abū Bakr dont le douzième chapitre est consacrée à ce genre de pratiques populaires. Ainsi- lit-on que pour l'infécondité chez les animaux on écrivait surate "Alā "Umrān" "la famille de 'Umran" sur un plateau de cuivre avec du safran ensuite en rincait l'écriture et on faisait boire la jument, puis arroser les flancs la tête et tout le corps avec, elle deviendra féconde avec la volonté de Dieu. A titre d'exemple pour éloigner le mauvais œil, le regard envieux et la colique obstructive de l'animal, on accrochait à son cou une amulette contenant un bout de cerf, un crin de cheval, et d'autres. ${ }^{81}$

Un second traité vétérinaire écrit à l'école al-Zāhirīya à Damas en 790h/1388, dont l'auteur est anonyme, rapporte que pour le soignement d'une bête malade on écrivait par terre certains versets coraniques et on faisait passer la bête au dessus. ${ }^{82}$

Il est bon de savoir que ces pratiques superstitieuses ont relevés les protestations de certains savants comme al-Subkī, qui qualifia ces pratiques d'innovations "bida'a", et que l'emploi des versets coraniques était inadmissible selon lui. ${ }^{83}$

\section{La valeur artistique}

Parlons maintenant du côté artistique. Quant à la repésentation des personages et des animaux elle est de profile. Ils sont peints à la gouache et rehaussés d'or. Les vétérinaires apparaissent le corps frêle, les épaules étroites, et la tête auréolée. Ces personages ont le nez croché, le menton rehaussé. Ils portent une robe courte sur un long pantalon. Par contre les animaux comme le cheval, le dromadaire et le taureau représentent le thème principal, ils sont grandement représentés par rapport aux vétérinaires. Certains chevaux sont des chevaux arabes d'autres sont

${ }^{81}$ Abū Bakr, Le traité, p.182; Nabīl, al-Hayl, p.84.

${ }^{82}$ Anomyme, al-Baīţara al-Rumīya, ms. 1334, Dār al-Kutub al-Masrīya, fol.15.

${ }^{83}$ Al-Subkī, Mu īid, pp.144-145; Nabīl, al-Hayl, p.122. 
petits et trapus et remsemblent aux mulets. La repésentation de scènes n'est pas pourtant totalement dépourvue de mouvements. ${ }^{84}$

Quant à la représentation végétale, elle est simplement incarnée, il s'agit de quelques végétaux éparpillés sur le terrain ou bien, on voit de petits arbres munis de fleurs. La présence de quelques pierres dénote un terrain rocailleux. L'écurie est repésentée simplement par une barre horizontale.

Suivant les chercheurs l'illustration de ce manuscrit rappelle l'école de Bağdād. ${ }^{85}$

Pour en conclure, on peut dire que les travaux vétérinaires à cette époque étaient diversifiés et riches en recettes médicales, autrement dit il y avait plusieurs traitements pour une maladie singulière. Le vétérinaire arabe maîtrisait bien son travail.

Quant aux remèdes employés certains étaient d'origine végétale comme l'aloés, l'huile d'olive, l'asphodèle. D'autres d'origine minérale comme les coquillages, la pierre ponce et le sel.

Suivant les manuscrits cités, durant certains soins chirurgicaux l'animal étaient ligoté, et non anesthésié, cependant le vétérinaire le faisait perdre connaissance dans quelques opérations majeures.

A côté d'un vétérinaire expérimenté, le palfrenier jouait un rôle non moins important.

On peut en conclure aussi que l'opération de la saignée était assez répandue à cette époque.

Suivant Mazahérī, outre que les vétérinaires spécialisés, il existait d'autres personnes qui pratiquaient légèrement l'hippiatrie comme les forgerons.

\footnotetext{
${ }^{84}$ Zakī Muhammad Hasan, Madrasat bag̉dād fĩ al-taşwīr al-islāmī, p.12; Sumar, XI, volume I, p.12.Hasan al-Bāšā, al-Taşwīr fĩ al- 'uşūr al-wūsțā, Dār al-Nahda al- Arabīya, 1992, p.139.

${ }^{85}$ J.Stchoukine, Les Manuscrits illustrés musulmans de la bibiothèque du Caire, Gazette des beaux arts, XII, 1935, pp.138-140; 'Abd al-Rāziq, al-Hadāra, p.236.
} 
$r$

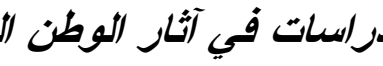

\section{Résumé}

Cette recherche a pout but de jetter la lumière sur la médecine vétérinaire arabe au moyen âge, depuis le VIième/XIIième siècle jusqu'au IXième/XVième siècle, autrement dit sous la période mamlūke. Celle-ci fut reconnue par les exercises militaires, les jeux sportifs et les cortèges.

Tout d'abord la recherche cite le nom de quelques vétérinaires célèbres, ensuite elle signale en bref, les maladies relatives à cette époque. En outre elle souligne les différentes méthodes curatives, à savoir trois méthodes:

a- Médecine préventive,comme l'hygiène y compris le nettoyage des animaux et des écuries, le nettoyage de la nourriture des déchets qui peuvent causer du mal à l'animal.Ceci était le travail du palfrenier.

b-Médecine curative comme l'embryotomie, la castration, la cautérisation,la luxation,l'utilisation des onguents, des collyres, de infusions, la fumigation, le lavage par voie anale et d'autres.Les vétérinaires arabe avaient recours aux produits minérales comme le coquillage,sel d'Andran.Ainsi que les produits végétales comme l'huile d'olive le vinaigre, l'eau de rose.

c- Médecine populaire, comme la suspension des amulettes, la guérison par le biais du coran, qui était pratiqué par certains vétérinaires ou palfreniers.

On conclut la diversifité des recettes curatives pour une seule maladie comme la castration, l'embryotomie. On note l'absence de l'anesthésie générale durant les soins chirurgicaux majeures comme l'opium, et pour remplacer, le vétérinaire faisait perdre connaissance à l'animal.

On peut dire aussi que la saignée était répandue quant à la guérison de plusieurs maladies et comme préventive.

A côté d'une médecine vétérinaire savante, il existait une médecine populaire basée sur les incantations par le biais des versets coraniques et d'autres comme les amulettes.

A cette période, en dépit des hippiatres il y avaient les forgerons qui pratiquaient légèrement la médecine vétérinaire. 


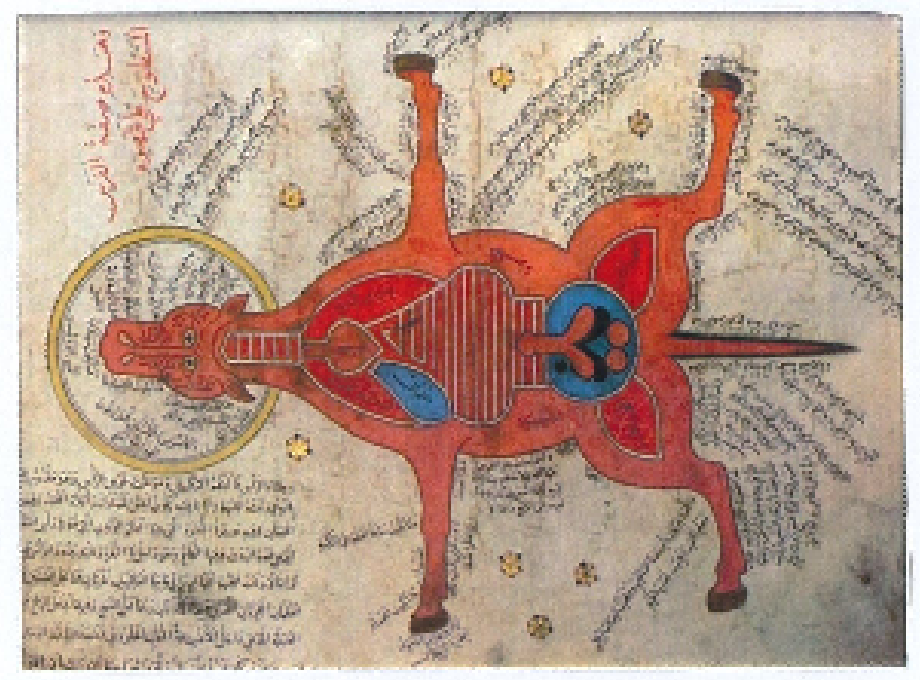

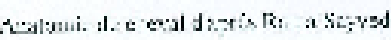

II.II

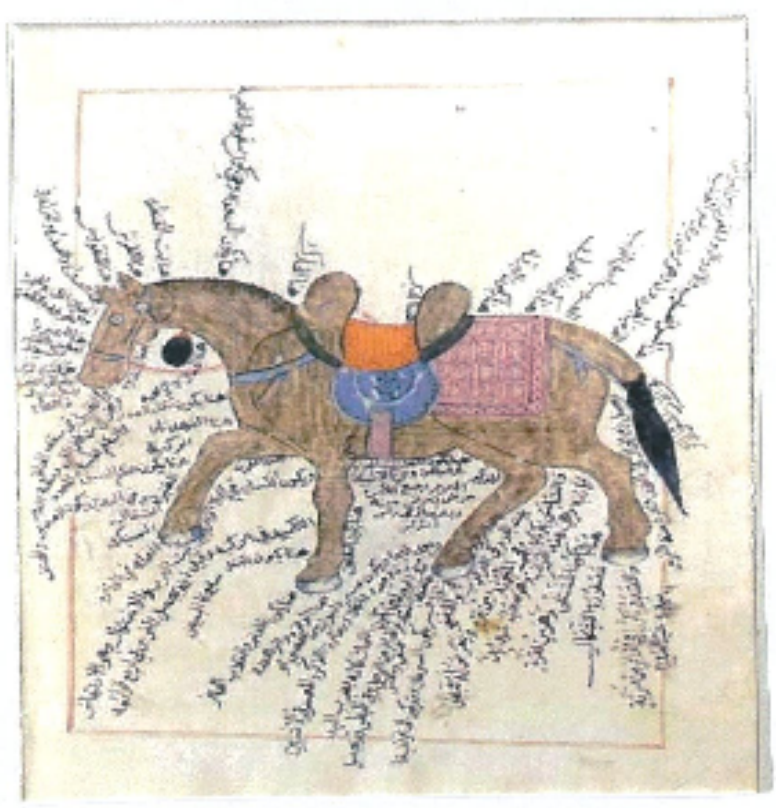

Malaties de cheral diapres Rurix Szyod 


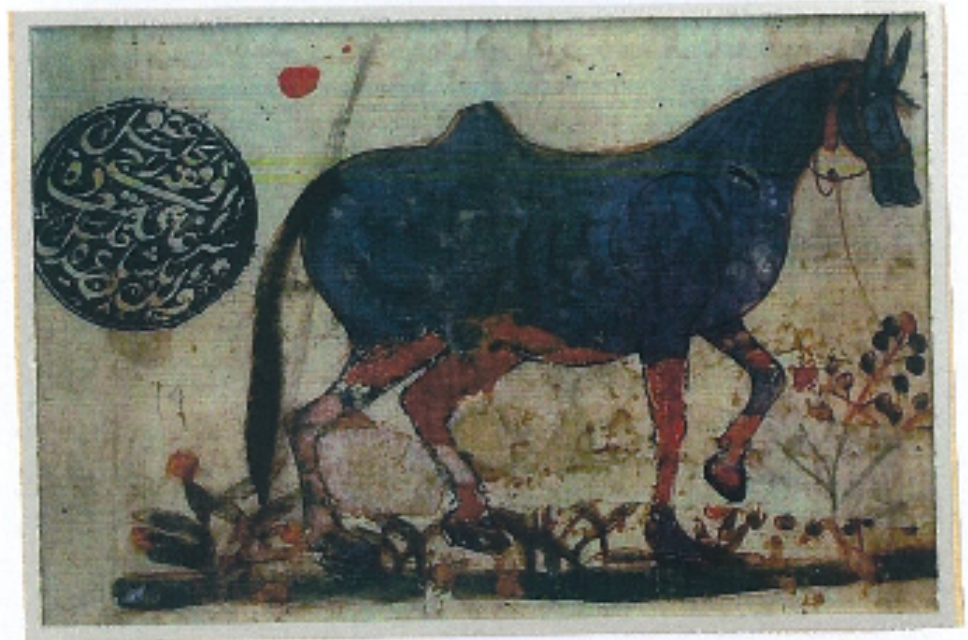

Cheral bossin, d'apres (land les scierees parknt aridic

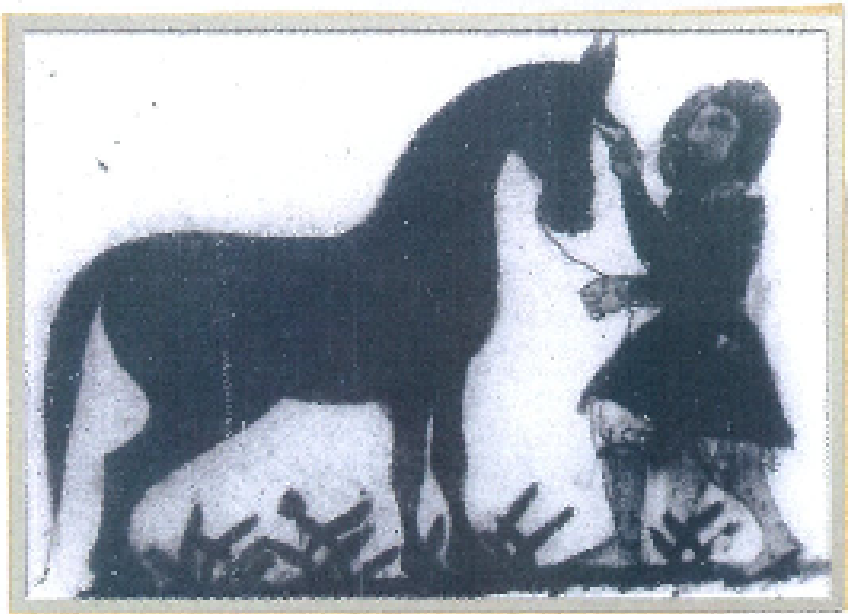

Lifplicuten te lasuriforten pur a vitemix Clapn's Il: sil-s.lu: 


\section{PI.V}

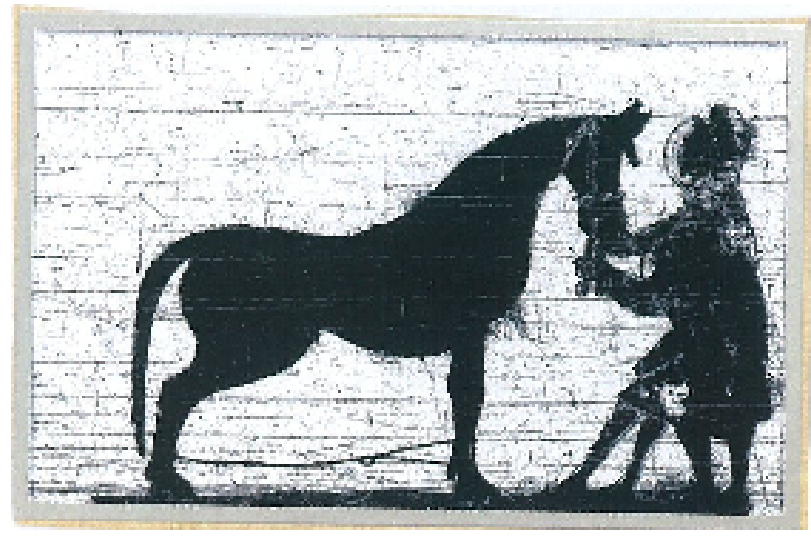

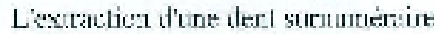
T)'a[rés Thu al-Alinaf

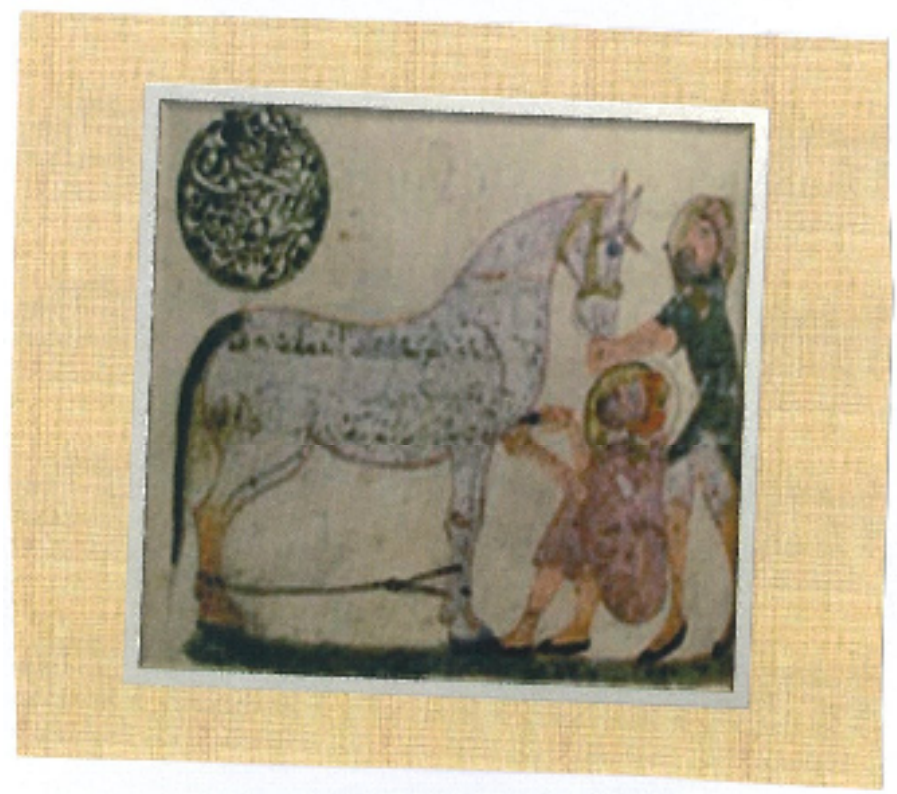

Deux xćtóriaaiıs appliquenu le

bistouri ct la fumigetion à ur cheval.

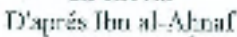


|P|W|

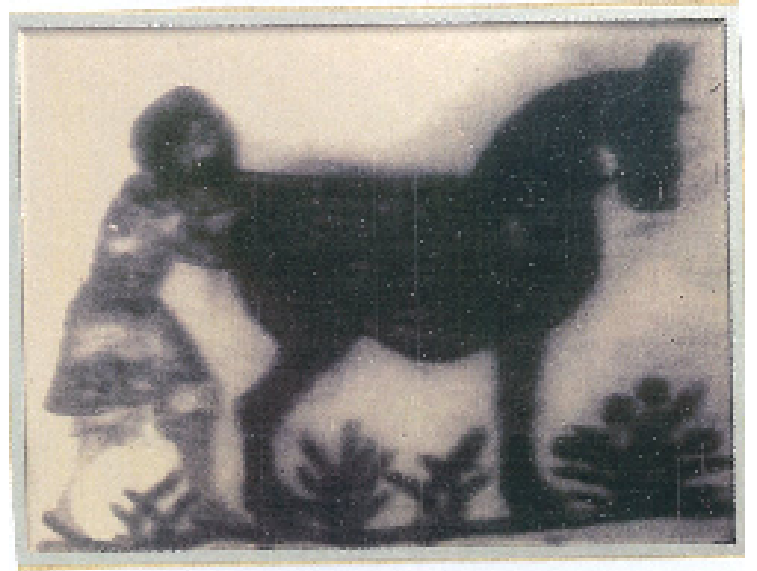

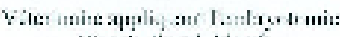
Ios th in: al that

[י. VIII

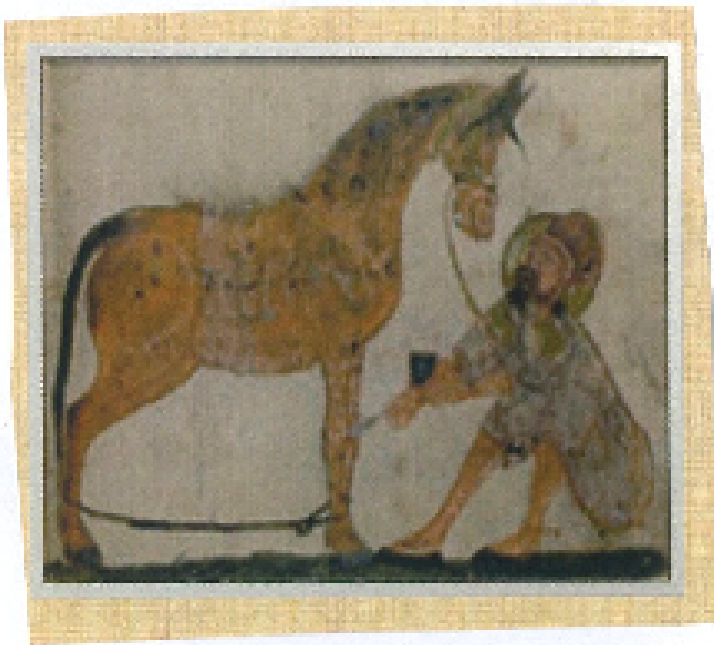

Viskir-dive appliatume ls cart:er:

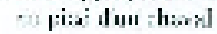

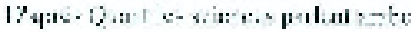




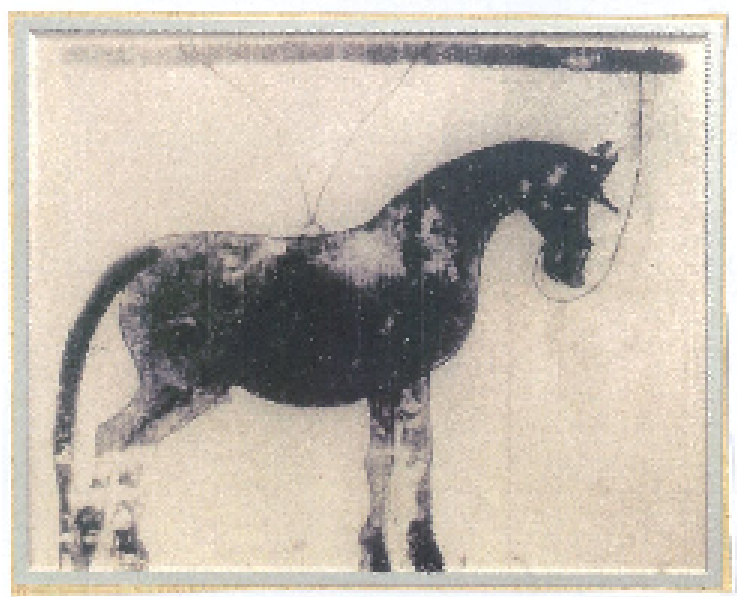

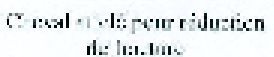

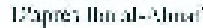

PIX

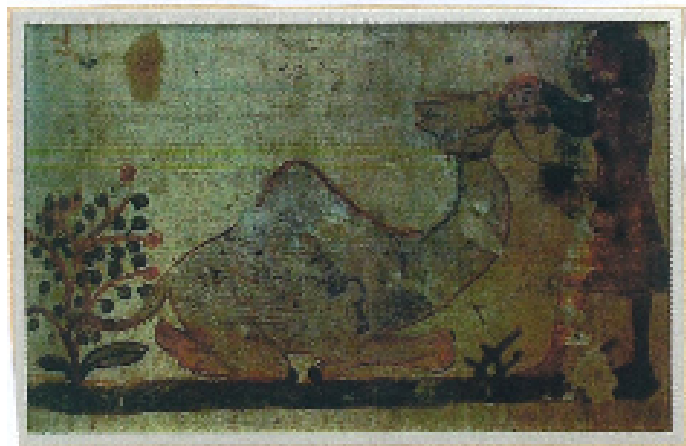

iscect:alce ch 'min c.: ju: i

$\checkmark$ thuts da c escin

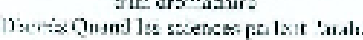


دراسات في آثار الوطن العربي ب 1

Pl.XI

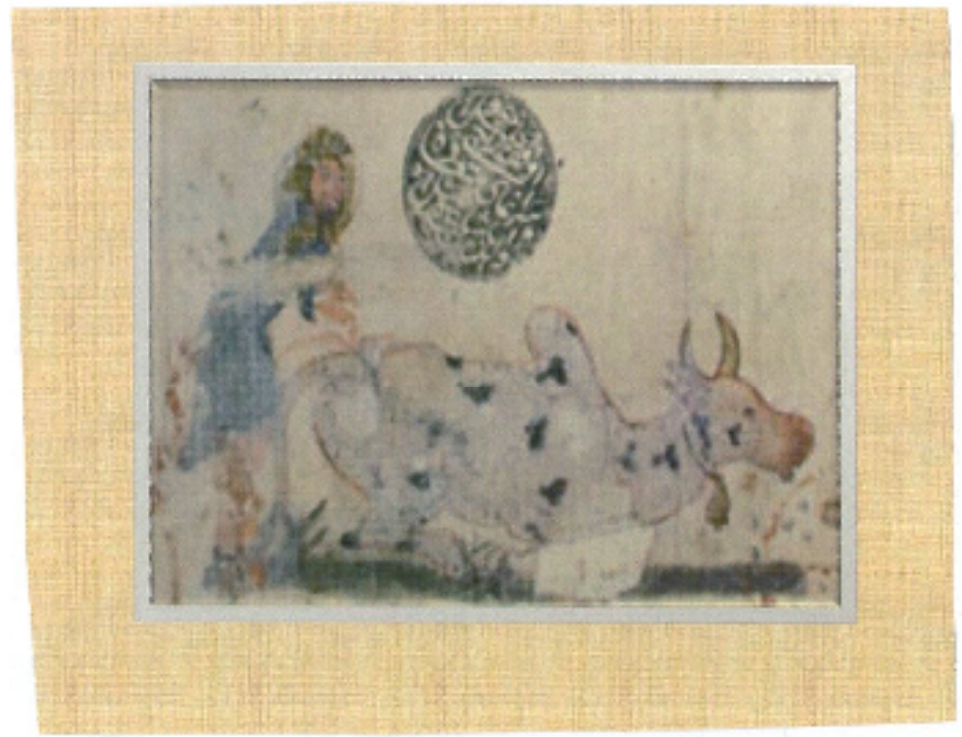

Vábinatice aui retucte de la force

à

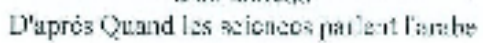




\section{• د ديهان حشمت}

الملخص

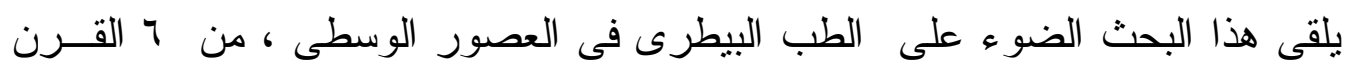

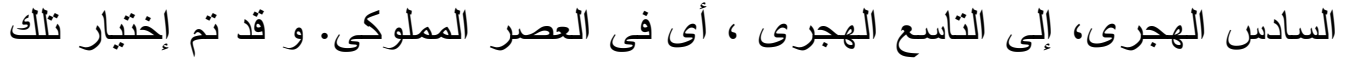

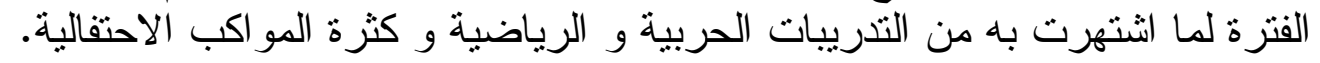

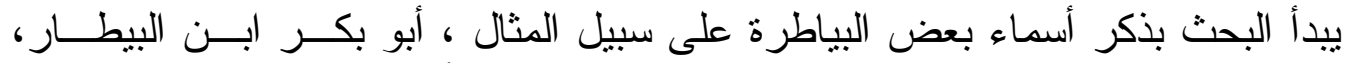

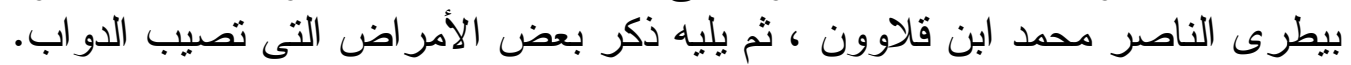

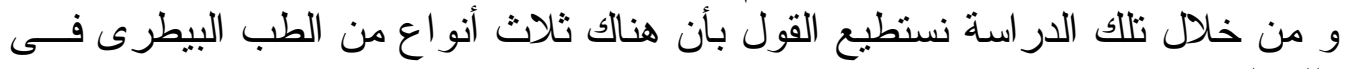

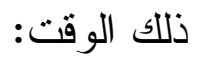
أ- الطب الوقائى، مثال مر اعاة نظافة الحظائر و الحيو انات و الاهتمام بطعام الحيوان

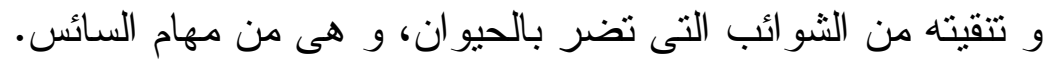

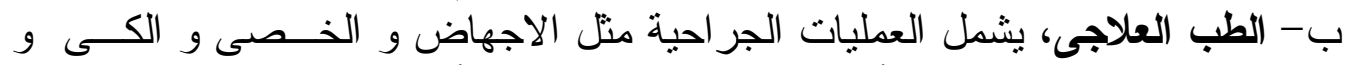

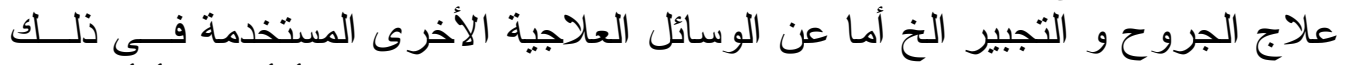

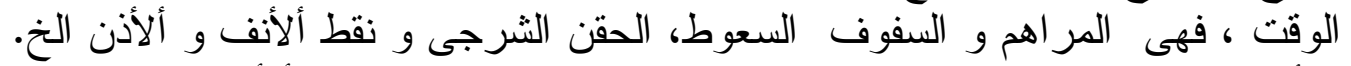

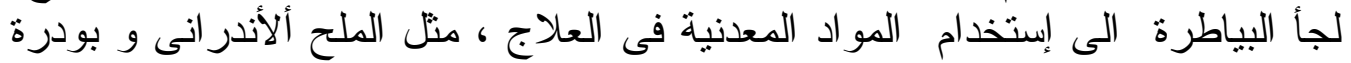

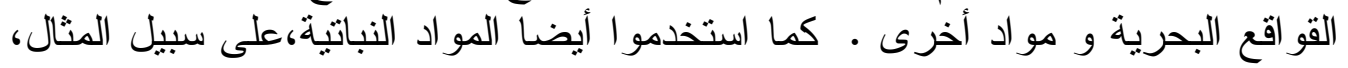

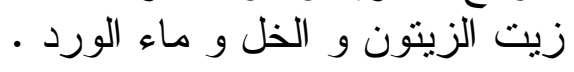

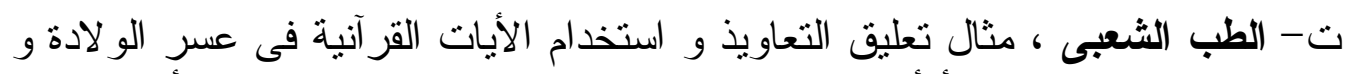

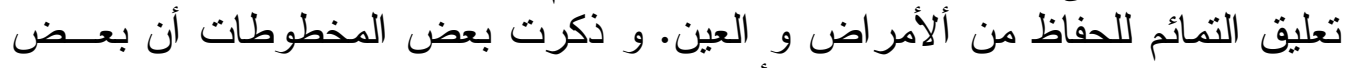

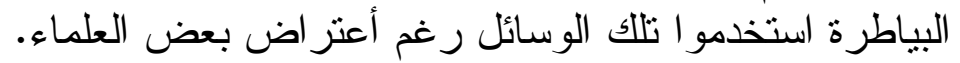

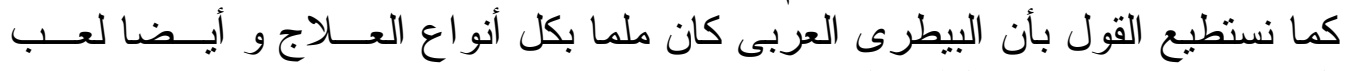

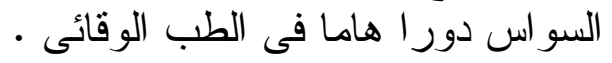

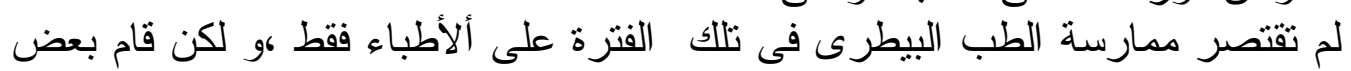

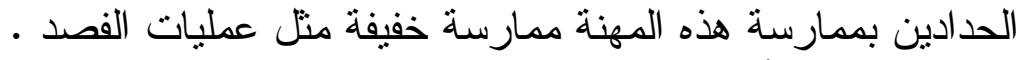

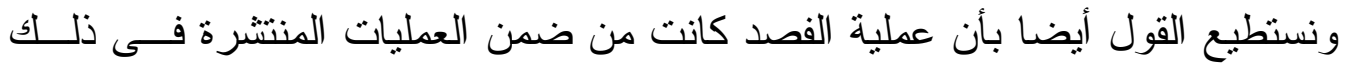

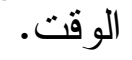

$$
\text { • مدرس - تخصص اسلامي - معهد السياحة و الفنادق - شير اتون - هيليوبوليس . }
$$

